\title{
Non-fluoroscopic navigation systems for radiofrequency catheter ablation for supraventricular tachycardia reduce ionising radiation exposure
}

\author{
Jason $\underline{\mathrm{See}}^{1}$, MBBS, MRCP, Jonah L $\underline{\text { Amora }}{ }^{2}$, MD, Sheldon $\underline{\mathrm{Lee}}^{1}$, MBBS, MRCP, Paul $\underline{\mathrm{Lim}}^{2}$, MBBS, MRPC, Wee Siong $\underline{\text { Teo }}{ }^{2}$, MBBS, FAMS,
} Boon Yew $\underline{\mathrm{Tan}}^{2}$, MBChB, FAMS, Kah Leng $\underline{\mathrm{Ho}}^{2}$, MBBS, MRCP, Chee Wan $\underline{\mathrm{Le}}^{3}$, MBBS, MRCP, Chi Keong $\underline{\mathrm{Ching}}{ }^{2}$, MBBS, FAMS

INTRODUCTION The use of non-fluoroscopic systems (NFS) to guide radiofrequency catheter ablation (RFCA) for the treatment of supraventricular tachycardia (SVT) is associated with lower radiation exposure. This study aimed to determine if NFS reduces fluoroscopy time, radiation dose and procedure time.

METHODS We prospectively enrolled patients undergoing RFCA for SVT. NFS included EnSite ${ }^{\mathrm{TM}} \mathrm{NavX}^{\mathrm{TM}}$ or CARTOß mapping. We compared procedure and fluoroscopy times, and radiation exposure between NFS and conventional fluoroscopy (CF) cohorts. Procedural success, complications and one-year success rates were reported.

RESULTS A total of 200 patients over 27 months were included and RFCA was guided by NFS for 79 patients; those with atrioventricular nodal reentrant tachycardia (AVNRT), left-sided atrioventricular reentrant tachycardia (AVRT) and right-sided AVRT were included ( $n=101,63$ and 36 , respectively). Fluoroscopy times were significantly lower with NFS than with CF $(10.8 \pm 11.1$ minutes vs. $32.0 \pm 27.5$ minutes; $p<0.001)$. The mean fluoroscopic dose area product was also significantly reduced with NFS (NSF: 5,382 $\pm 5,768 \mathrm{mGy}^{*} \mathrm{~cm}^{2}$ vs. CF: $21,070 \pm 23,311 \mathrm{mGy}^{*} \mathrm{~cm}^{2} ; \mathrm{p}<0.001$ ); for all SVT subtypes. There was no significant reduction in procedure time, except for left-sided AVRT ablation (NFS: 79.2 minutes vs. $C F: 116.4$ minutes; $p=0.001$ ). Procedural success rates were comparable (NFS: $97.5 \%$ vs. CF: $98.3 \%$ ) and at one-year follow-up, there was no significant difference in the recurrence rates (NFS: $5.2 \%$ vs. CF: $4.2 \%$ ). No clinically significant complications were observed in both groups.

CONCLUSION The use of NFS for RFCA for SVT is safe, with significantly reduced radiation dose and fluoroscopy time.

Keywords: non-fluoroscopic systems, radiofrequency catheter ablation, supraventricular tachycardia

\section{INTRODUCTION}

Radiofrequency catheter ablation (RFCA) is a first-line therapeutic option for supraventricular tachycardia (SVT). ${ }^{(1)}$ It is conventionally performed under fluoroscopy. The amount of exposure to fluoroscopy is highly dependent on multiple variables, such as operator experience and the complexity of the arrhythmia substrate. ${ }^{(2)}$ As exposure to radiation during such procedures increases the lifetime risk of fatal malignancies, skin injuries and cataract, it poses a palpable hazard to both patients and medical staff. ${ }^{(3)}$ This emphasises the importance of employing all possible measures to minimise exposure to ionising radiation.

Recent advances in technology have led to the development of non-fluoroscopic three-dimensional (3D) mapping systems to guide ablation during prolonged electrophysiological procedures, such as pulmonary vein isolation for atrial fibrillation and mapping of atypical atrial flutter and ventricular tachycardia. ${ }^{(4)}$ These 3D mapping systems help physicians to better comprehend complex arrhythmias and develop appropriate ablation strategies. These systems have also been proven to substantially reduce ionising radiation exposure. ${ }^{(5-7)}$

Data on the use of non-fluoroscopic systems (NFS) in Singapore is limited. Therefore, the aim of the present study was to determine the consistency and applicability of the aforementioned latest findings in our local setting. The specific aims of the present study were (a) to determine the procedure and fluoroscopy times, and ionising radiation exposure of patients who underwent RFCA for SVT using NFS as compared to those using conventional fluoroscopy (CF); and (b) to compare atrioventricular nodal reentrant tachycardia (AVNRT) ablation and atrioventricular reentrant tachycardia (AVRT) ablation.

\section{METHODS}

The present study was a prospective analysis of patients who underwent electrophysiology (EP) study of paroxysmal SVT at our institution from January 2012 to March 2014. Patients with AVNRT and AVRT were enrolled and a total of 200 consecutive patients, aged 11-86 years, who underwent RFCA were included in this study. All patients (or parents, if the patient was underage) gave informed consent.

Each patient underwent RFCA in the fasted and non-absorptive state, and under intravenous sedation with midazolam and fentanyl. Local anaesthetic with lignocaine was administered at the vascular access sites, which were the femoral venous site for all patients. Some patients also had femoral arterial access if the operator selected a retrograde aortic approach to obtain access to the left side of the heart. The decision for NFS usage was operator-dependent and made before the EP study. The choice of NFS, either EnSite ${ }^{\mathrm{TM}} \mathrm{NavX}^{\mathrm{TM}}$ (St Jude Medical, St Paul, MN, USA)

${ }^{1}$ Department of Cardiology, Changi General Hospital, ${ }^{2}$ Department of Cardiology, National Heart Centre Singapore, ${ }^{3}$ Department of Cardiology, Khoo Teck Puat Hospital, Singapore Correspondence: A/Prof Ching Chi Keong, Senior Consultant, Department of Cardiology, National Heart Centre Singapore, 5 Hospital Drive, Singapore 169609. Ching.Chi.Keong@nhcs.com.sg 

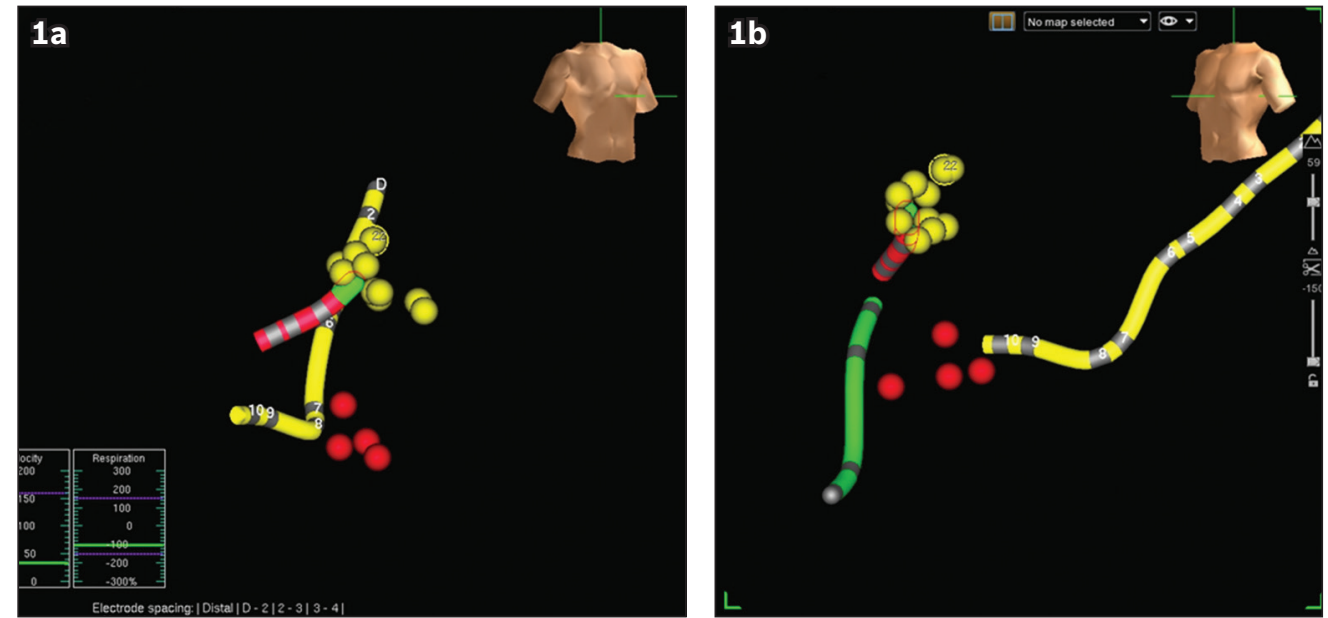

Fig. 1 Images show NavX mapping of the His region and the slow atrioventricular (AV) node pathway, in (a) right anterior oblique and (b) left anterior oblique views of the NavX screen during ablation of atrioventricular nodal reentrant tachycardia. The yellow dots show the region where His potentials were recorded and tagged. The red dots depict the regions where radiofrequency energy was applied and junctional rhythm was subsequently recorded; these regions were inferred to be the slow pathway region of the AV node. The slow pathway region of the AV node is therefore just anterior to the ostium of the coronary sinus (yellow tube), and inferior and slightly more medial to the His region. Note the safe distance between the yellow and red dots. A quadpolar catheter was placed in the right ventricular apex (green tube).

or CARTO ${ }^{\circledR}$ (Biosense Webster Inc, Diamond Bar, CA, USA), was also made according to the operator's preference. In brief, the EnSite NavX system uses impedance measurements between individual catheter electrodes and external patches placed on the chest to project a 3D image of the catheters, while CARTO uses magnetic technology to triangulate the location and orientation of a specialised catheter equipped with a magnet sensor on its tip. For both systems, three pairs of orthogonal skin patches were applied over the chest and on the back, in the usual recommended positions. Either the coronary sinus catheter or an external patch was selected as the reference.

Fluoroscopy was performed using Philips FD10 (Philips Electronics North America, Andover, MA, USA). The minimum dose compatible with adequate imaging was used during the advancement of the catheters into the conventional locations and for confirmation of the catheter positions. Once suitable His signals were acquired, the area was tagged on the mapping screen (Fig. 1). The CardioLab EP recording system (GE Healthcare, Cleveland, OH, USA) was utilised for all EP studies. Standard protocols and procedures, depending on the arrhythmic substrate, were used for all ablation procedures.

Procedure time (in minutes) was defined as the time interval from the initial access site puncture to removal of all catheters. Fluoroscopy time (in minutes) was defined as the cumulative duration of fluoroscopy during the entire procedure. Radiation dose (in $\mathrm{mGy}^{*} \mathrm{~cm}^{2}$ ) was the calculated dose area product (DAP) for the specific patient. Procedural success for AVNRT was defined as the absence of inducible tachycardia > 20 minutes after the last RFCA application and the presence of up to a single atrioventricular nodal echo with intravenous isoprenaline challenge. Procedural success for AVRT was defined as the noninducibility of tachycardia, loss of pre-excitation (if manifested) and loss of retrograde accessory pathway conduction after 20 minutes of observation following the last applied RFCA.

All patients were monitored with telemetry for at least one night. Patients were all scheduled for outpatient visits within four months of discharge and were followed up for at least 12 months. Data analysis was performed using SPSS version 16.0 (SPSS Inc, Chicago, IL, USA). Continuous variables were expressed as mean values and compared using Student's $t$-test, while categorical data was analysed using chi-square test. A (two-tailed) p-value $<0.05$ was considered statistically significant.

\section{RESULTS}

Among the 200 consecutive patients who underwent RFCA, NFS was used for 79 patients, while CF was used for 121 patients (Table I). The mean age of patients who underwent RFCA with NFS (i.e. NFS group) was $39.5 \pm 16.3$ years, while the mean age of patients who underwent RFCA with CF (i.e. CF group) was $43.4 \pm 17.9$ years. Among the patients in the NFS group, 35 (44.3\%) had AVNRT, 25 (31.6\%) had left-sided AVRT and $19(24.1 \%)$ had right-sided AVRT. Among the patients in the CF group, 66 (54.5\%) had AVNRT, 38 (31.4\%) had left-sided AVRT and $17(14.0 \%)$ had right-sided AVRT. There was no significant difference in the baseline demographics of the two groups.

The mean procedure time was $87.8 \pm 43.5$ minutes for the NFS group and $99.7 \pm 50.4$ minutes for the CF group ( $p=0.088)$. When this was analysed according to the type of SVT, we found that the mean procedure time for left-sided AVRT ablation was lower among patients in the NFS group than the CF group $(79.2 \pm 29.4$ minutes vs. $116.4 \pm 58.2$ minutes; $p=0.001)$ (Table II). The mean procedure time of patients who had NFS and CF were not significantly different among patients with AVNRT ablation ( $p=0.802$ ) and right-sided AVRT ablation ( $p=0.424)$.

The mean fluoroscopy time of patients in the NFS group was significantly lower than that of patients in the CF group (10.8 \pm 11.1 minutes vs. $32.0 \pm 27.5$ minutes; $p<0.001)$. Among the patients with AVNRT, the mean fluoroscopy time of patients in the NFS group was significantly lower than that of the CF group $(6.8 \pm 5.8$ minutes vs. $20.3 \pm 10.6$ minutes; $p<0.001)$ (Table II). This trend was consistent among patients with left-sided AVRT (NFS: $14.1 \pm 11.7$ minutes vs. CF: $42.9 \pm 30.2$ minutes; $p<0.001$ ) 
and right-sided AVRT (NFS: $14.0 \pm 15.1$ minutes vs. CF: $53.0 \pm$ 42.3 minutes; $\mathrm{p}=0.002$ ).

The mean fluoroscopic DAP of patients in the NFS group was significantly lower than that of the CF group $\left(5,382 \pm 5,768 \mathrm{mGy}^{*} \mathrm{~cm}^{2}\right.$ vs. 21,070 $\pm 23,311 \mathrm{mGy}^{*} \mathrm{~cm}^{2}$; $\mathrm{p}<0.001$ ). Among the patients with AVNRT, the mean radiation dose received by patients in the NFS group was significantly lower than that received by the CF group $\left(3,920 \pm 4,625 \mathrm{mGy}^{*} \mathrm{~cm}^{2}\right.$ vs. $13,619 \pm 9,769 \mathrm{mGy}^{*} \mathrm{~cm}^{2} ; \mathrm{p}<0.001$ ) (Table II). This trend was also noted among patients with left-sided AVRT (NFS: 6,561 \pm 6,207 mGy* $\mathrm{cm}^{2}$ vs. CF: 25,518 $\pm 19,606 \mathrm{mGy}^{*} \mathrm{~cm}^{2} ; \mathrm{p}<0.001$ ) and right-sided AVRT (NFS: 6,526 $\pm 6,703 \mathrm{mGy}^{*} \mathrm{~cm}^{2}$ vs. CF: 40,322 $\left.\pm 46,047 \mathrm{mGy}^{*} \mathrm{~cm}^{2} ; \mathrm{p}=0.008\right)$.

Overall, acute procedural success rates were comparable between the NFS and CF groups (97.5\% [77/79] vs. $98.3 \%$ [119/121]; $p=0.51)$. The acute procedural success rates for AVNRT ablation and left-sided AVRT ablation were both $100 \%$ in the NFS and CF groups. Four patients with right-sided AVRT did not achieve procedural success - two underwent NFS and two underwent CF. The overall acute procedural success rate of

Table I. Baseline and clinical characteristics of the study population ( $n=200$ ), according to the method used for radiofrequency catheter ablation.

\begin{tabular}{lccc}
\hline Characteristic & \multicolumn{2}{c}{ No. (\%) } & p-value \\
\cline { 2 - 3 } & NFS (n=79) & CF (n = 121) & \\
\hline Age* (yr) & $39.5 \pm 16.3$ & $43.4 \pm 17.9$ & 0.119 \\
Gender & $41(51.9)$ & $59(48.8)$ & 0.772 \\
Male & $38(48.1)$ & $62(51.2)$ & \\
Female & & & \\
Comorbidity & $12(15.2)$ & $21(17.4)$ & 0.846 \\
Hypertension & $5(6.3)$ & $16(13.2)$ & 0.158 \\
Diabetes mellitus & $14(17.7)$ & $29(24.0)$ & 0.379 \\
Hyperlipidaemia & $4(5.1)$ & $7(5.8)$ & 1.000 \\
Ischaemic heart & & & \\
disease & $35(44.3)$ & $66(54.5)$ & 0.051 \\
Type of SVT & $19(24.1)$ & $17(14.0)$ & $<0.001$ \\
AVNRT & $25(31.6)$ & $38(31.4)$ & 0.960 \\
Right-sided AVRT & & & \\
Left-sided AVRT & & & \\
\hline
\end{tabular}

*Data presented as mean \pm standard deviation. AVNRT: atrioventricular nodal reentrant tachycardia; AVRT: atrioventricular reentrant tachycardia; CF: conventional fluoroscopic; NFS: non-fluoroscopic systems; SVT: supraventricular tachycardia patients with right-sided AVRT from the NFS group was $89.5 \%$ (17/19); two patients who did not achieve procedural success had para-Hisian accessory pathways and repeated ablations were not pursued. In comparison, the acute procedural success rate of patients with right-sided AVRT from the CF group was $88.2 \%$ (15/17); two patients who had failed ablation were diagnosed with Ebstein's anomaly and had multiple accessory pathways.

No clinically significant periprocedural complications (e.g. atrioventricular block, cardiac tamponade and periprocedural myocardial infarction) were observed in both the NFS and CF groups. All patients were discharged from the hospital uneventfully. Among the patients who had acute procedural success, $5.2 \%$ (4/77) of patients from the NFS group and $4.2 \%$ (5/119) from the CF group had arrhythmia recurrence within the 12 months of follow-up; this was not statistically significant $(p=0.50)$.

\section{DISCUSSION}

Our study demonstrated that the use of NFS during RFCA for SVT resulted in significant reductions in ionising radiation exposure with no impact on overall procedure time, except for patients with leftsided AVRT (for which procedure time was significantly lower for the NFS group than the CF group). The overall results of our study are comparable to the only randomised trial of NFS, which was conducted by Earley et al, ${ }^{(8)}$ they reported a median fluoroscopy time of six minutes for CARTO and four minutes for EnSite NavX. In our study, the fluoroscopy time of the NFS group was significantly lower than that of the CF group $(10.8 \pm 11.1$ minutes vs. $32.0 \pm$ 27.5 minutes; $p<0.001)$. Earley et al also reported a median DAP value of 5,000 $\mathrm{mGy}^{*} \mathrm{~cm}^{2}$ for CARTO and 2,000 $\mathrm{mGy}^{*} \mathrm{~cm}^{2}$ for EnSite NavX ${ }^{(8)}$ the mean DAP value for NFS in the present study $\left(5,382 \pm 5,768 \mathrm{mGy}^{*} \mathrm{~cm}^{2}\right)$ approximated these median CARTO values. Variations in the numbers may be due to the different methodologies used. It is unclear whether Earley et al utilised fluoroscopy in the placement of catheters prior to using NFS. ${ }^{(8)}$ Furthermore, their study population included patients with atrial flutter ablations (about $20 \%$ of the study cohort); ${ }^{(8)}$ such patients were excluded in the present study's analysis.

Among the patients with AVNRT who underwent RFCA $(n=101)$, there was a significant 13.5-minute absolute reduction in the mean total fluoroscopy time of the NFS group compared to the CF group (NFS: $6.8 \pm 5.8$ minutes vs. CF: $20.3 \pm 10.6$ minutes; $\mathrm{p}<0.001)$. This mirrors the results of a small, randomised, single-

Table II. Fluoroscopy time, radiation dose and procedure time of radiofrequency catheter ablation, according to the type of supraventricular tachycardia.

\begin{tabular}{|c|c|c|c|c|c|c|c|c|c|}
\hline \multirow[t]{2}{*}{ Variable } & \multicolumn{3}{|c|}{ AVNRT } & \multicolumn{3}{|c|}{ Right-sided AVRT } & \multicolumn{3}{|c|}{ Left-sided AVRT } \\
\hline & NFS & CF & p-value & NFS & CF & p-value & NFS & CF & p-value \\
\hline $\begin{array}{l}\text { Fluoroscopy } \\
\text { time (min) }\end{array}$ & $6.8 \pm 5.8$ & $20.3 \pm 10.6$ & $<0.001$ & $14.0 \pm 15.1$ & $53.0 \pm 42.3$ & 0.002 & $14.1 \pm 11.7$ & $42.9 \pm 30.2$ & $<0.001$ \\
\hline $\begin{array}{l}\text { Radiation dose } \\
\left(m G{ }^{*} \mathrm{~cm}^{2}\right)\end{array}$ & $3,920 \pm 4,625$ & $13,619 \pm 9,769$ & $<0.001$ & $6,526 \pm 6,703$ & $40,322 \pm 46,047$ & 0.008 & $6,561 \pm 6,207$ & $25,518 \pm 19,606$ & $<0.001$ \\
\hline $\begin{array}{l}\text { Procedure time } \\
(\min )\end{array}$ & $80.7 \pm 41.7$ & $82.5 \pm 30.1$ & 0.802 & $112.3 \pm 53.9$ & $128.9 \pm 69.0$ & 0.424 & $79.2 \pm 29.4$ & $116.4 \pm 58.2$ & 0.001 \\
\hline
\end{tabular}

Data presented as mean \pm standard deviation. AVNRT: atrioventricular nodal reentrant tachycardia; AVRT: atrioventricular reentrant tachycardia; CF: conventional fluoroscopy; NFS: non-fluoroscopic systems 
operator study by Kopelman et al, which reported a significant absolute reduction in the mean total fluoroscopy exposure by 11.7 minutes. ${ }^{(9)}$ The substantial drop in fluoroscopy time may be attributed to the capability of NFS to accurately map the extent of the His region and display the location of the coronary sinus catheter (and the coronary sinus ostium) (Fig. 1). NFS provides a visual of the area of the slow pathway, illustrating the safe distance from the His region for ablation. Hence, there is less reliance on fluoroscopy to assess the position of the ablation catheter, so as to avoid inadvertent atrioventricular block during RFCA. Similarly, Kopelman et al also quantified the use of fluoroscopy during the application of RFCA and found a consistent reduction in fluoroscopy time when NFS was used. ${ }^{(9)}$ Importantly, the success rate when NFS was used was equal to that when CF was used in the present study. While we did not measure fluoroscopy duration during the application of RFCA, the protocol used in the present study was similar to that used by Kopelman et $\mathrm{al}^{\left({ }^{(9)}\right.}$ in that the use of NFS usually only commenced once all the catheters were positioned with fluoroscopic guidance.

The mean radiation dose was significantly reduced in patients with AVNRT who underwent NFS as compared to those who underwent CF $\left(3,920 \pm 4,625 \mathrm{mGy}^{*} \mathrm{Cm}^{2}\right.$ vs. 13,619 \pm 9,769 $\left.\mathrm{mGy}^{*} \mathrm{Cm}^{2} ; \mathrm{p}<0.001\right)$. This finding echoes that of a small casecontrolled study conducted by Kirchhof et al, which examined the effects of the use of NFS for RFCA of several types of SVT. ${ }^{(10)}$ In that study, only patients with AVNRT had a significant reduction in the radiation dose received. Although that study used a different NFS (i.e. LocaLisa), its finding of a significant reduction in the radiation dose among patients with AVNRT is confirmed by the results of the present study. The methodology of the present study is also similar to that of Kirchhof et al's study. In both studies, fluoroscopy was utilised in the initial placement of the catheters and for monitoring their position during RFCA application; however, the radiation dose for patients with AVNRT who underwent NFS was substantially lower in that study $\left(189 \pm 58 \mathrm{mGy}^{*} \mathrm{~cm}^{2}\right)^{(10)}$ than in the present study. This marked difference may be due to Kirchhof et al's use of biplane fluoroscopy and their study's smaller sample size $\left(\mathrm{n}=15\right.$ for AVNRT). ${ }^{(10)}$

Procedural time was not significantly reduced by the use of NFS among AVNRT patients in the present study. This is congruent with a prospective study conducted by Alvarez et al, which involved 100 patients with AVNRT.(11) In Alvarez et al's study design, the use of fluoroscopy was totally avoided for half of their study population. Despite this, they did not observe a significant difference in the total procedure time, time spent for EP study (diagnosis time) and ablation time. This may be because procedure time encompasses the time spent diagnosing tachycardia using conventional EP study protocols; therefore, it is likely to be influenced by the complexity of the arrhythmia substrate. The use of NFS may therefore not meaningfully reduce the total procedure time in AVNRT ablation. Regardless, our finding that the procedure time was neither prolonged nor shortened by the use of NFS is encouraging; it highlights the utility of NFS as a guiding tool that is unlikely to significantly prolong procedure time even if time is consumed for mapping.
Among the patients with AVRT, ionising radiation exposure was likewise significantly reduced with the use of NFS. Among the patients with right-sided AVRT, NFS reduced fluoroscopy time (NFS: $14.0 \pm 15.1$ minutes vs. CF: $53.0 \pm 42.3$ minutes) and radiation dose (NFS: 6,526 $\pm 6,703 \mathrm{mGy}^{*} \mathrm{Cm}^{2}$ vs. 40,322 $\pm 46,047$ $\mathrm{mGy}{ }^{*} \mathrm{~cm}^{2}$ ). A similar effect was noted among the patients with left-sided AVRT (for fluoroscopy time, NFS: $14.1 \pm 11.7$ minutes, CF: $42.9 \pm 30.2$ minutes, for radiation dose, NFS: 6,561 $\pm 6,207$ $\mathrm{mGy}^{*} \mathrm{Cm}^{2}$, CF: 25,518 $\pm 19,606 \mathrm{mGy}^{*} \mathrm{~cm}^{2}$ ) (Table II). This finding is also in concordance with other studies, although a much larger absolute decrease in fluoroscopy time was demonstrated in the present study. In a large series by Kesek et al, ${ }^{(12)}$ the mean absolute reduction in fluoroscopy time was only four minutes, although it was statistically significant. In that study, however, the authors attributed the reduction to an improvement in the overall ablation technique rather than utilisation of NFS. ${ }^{(12)}$

In the present study, there was a significant decrease in procedure time between NFS and CF only among patients with left-sided AVRT. However, it is difficult to determine how much of the decrease in procedure time is attributable to the reduction in fluoroscopy time due to the use of NFS. Previous studies did not demonstrate any significant change in procedure time for patients with AVRT, although this subgroup of patients comprised only a small percentage of those study populations. ${ }^{(8,10,12)}$ We postulate that the reduction in the procedure time observed in the present study may be due to the reduced ablation time that resulted from enhanced guidance during mapping via NFS. Enhanced guidance enables the operator to pinpoint critical sites for ablation more accurately. Moreover, the location of the accessory pathway can be marked on the reconstructed geometry using NFS (Figs. 2 \& 3). Subsequent radiofrequency pulses can then be delivered in a precise manner, even if pre-excitation is minimised or transiently disappears after the initial RFCA delivery. ${ }^{(13)}$ However, we did not quantify the ablation time or the number of radiofrequency pulses delivered in the present study. Availability of such information would have helped support the aforementioned argument. In addition, the proposed explanation does not account for the fact that there was no difference in the procedure times of patients who underwent NFS and patients who underwent CF for right-sided AVRT in the present study. Other variables, such as the use of transseptal access for left-sided AVRT ablation, could have confounded our results.

Two types of NFS (EnSite NavX and CARTO) from different manufacturers were used in the present study. In the only prospective trial that directly compared these two systems in patients with SVT, the EnSite NavX system demonstrated greater reduction in the amount of radiation, in terms of both fluoroscopy time and DAP. ${ }^{(12)}$ Since the magnitude of the effect of each system was not distinguished in the present study, we were not able to single out any particular advantage of either system. We did not encounter any significant complication associated with the use of either system.

Numerous studies have reported the unwanted effects of radiation exposure on both patients and medical staff, particularly effects experienced in an invasive catheterisation laboratory. ${ }^{(14-16)}$ We can indirectly estimate the effective radiation doses for the 

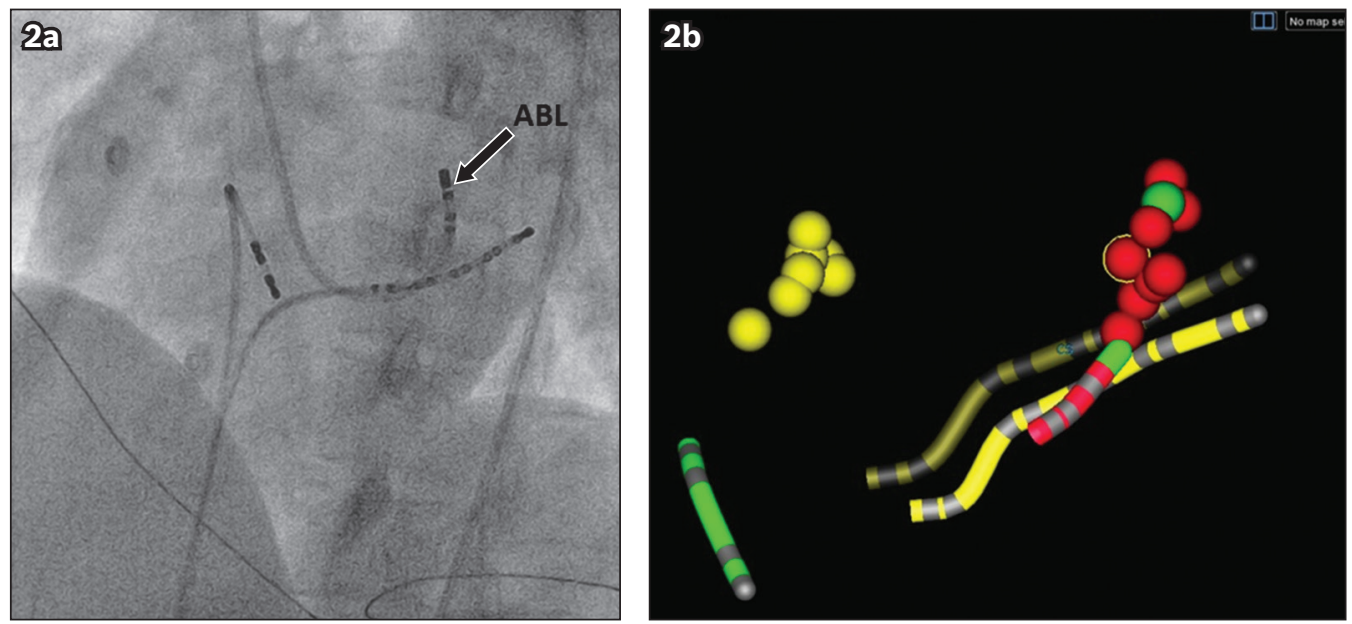

Fig. 2 (a) Fluoroscopic and (b) EnSite NavX images show the left anterior oblique view of the heart. The correlation between the fluoroscopic and EnSite NavX images of left-sided free wall accessory pathway ablation can be seen. On the fluoroscopic image, the ablation catheter (arrow) is seen at around the '3 o'clock' position. On the EnSite NavX image, the red dots represent the areas where radiofrequency catheter ablation has been perfomed; these areas correlate with the ablation catheter position on fluoroscopy. The green dot shows the earliest atrial electrogram during mapping. Note the safe distance between the His area (yellow dots) and the area of ablation. A decapolar catheter (yellow tube) was placed within the coronary sinus while a quadpolar catheter was placed in the right ventricular apex (green tube).
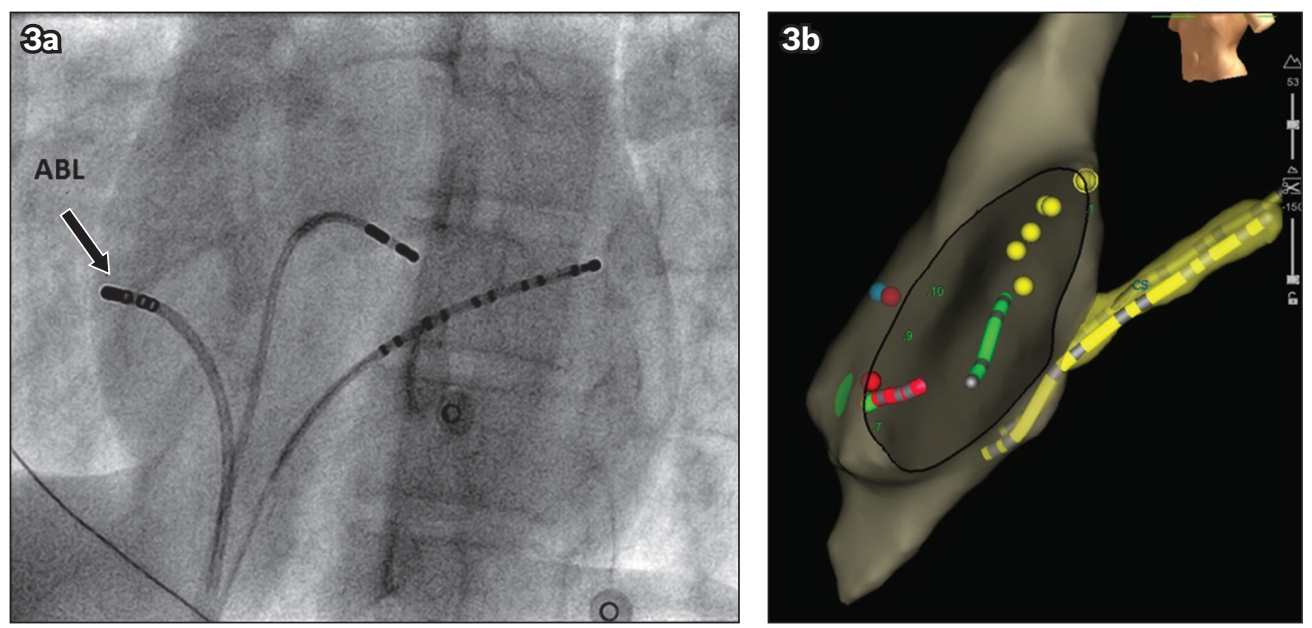

Fig. 3 (a) Fluoroscopic and (b) EnSite NavX images show the left anterior oblique view of the heart. The correlation between the fluoroscopic and EnSite NavX images of right-sided free wall accessory pathway ablation can be seen. On the fluoroscopic image, the ablation catheter (arrow) is seen near the ' 9 o'clock' position. On the EnSite NavX image, the red dots represent areas where radiofrequency energy has been applied. These areas correlate with the ablation catheter position on fluoroscopy. A decapolar catheter (yellow tube) was placed within the coronary sinus while a quadpolar catheter was placed in the right ventricular apex (green tube).

present study by converting the DAP values in the present study using the recommended conversion factor for cardiac ablation procedures. ${ }^{(17)}$ When this conversion was done, we obtained an estimated effective radiation dose of 3.1 millisieverts $(\mathrm{mSv})$ for patients with AVNRT who underwent ablation using CF. This roughly approximates to the value reported by Earley et al for the same subgroup of patients $(2.9 \mathrm{mSv}$, which translates to a lifetime risk of malignancy of 1:8,700). ${ }^{(8)}$ Earley et al reported that the lifetime risk of malignancy can be halved with the use of EnSite NavX for patients undergoing AVNRT ablation (the effective radiation dose was $1.3 \mathrm{mSv}$ for this cohort). ${ }^{(8)}$ In comparison, the estimated effective radiation dose among patients who underwent AVNRT ablation in the present study was lower $(0.90 \mathrm{mSv})$; this would be extrapolated to a lower lifetime risk of malignancy. Although these estimates are difficult to objectively quantify and interpret, any effective method to minimise radiation exposure is likely beneficial for both patients and medical staff. ${ }^{(13,14)}$ Such considerations are important, especially when the advantages of NFS are weighed against its apparent excessive cost.

The present study was not without limitations. The main limitation was that the study was non-randomised. The decision to use NFS was left entirely to the operator. Also, inter-operator variability in terms of skills and technique could not be controlled. Moreover, physicians who were under training participated, to some extent, in all of the institution's ablation procedures, with the possibility of greater exposure to conventional ablations (i.e. ablations using CF). We did not account for any difference that could have been caused by this factor. Nonetheless, we believe that the results of the present study are valid in a real-world setting, i.e. a training centre. A randomised prospective trial should be conducted to further evaluate the advantages of NFS over CF.

In conclusion, the use of NFS for RFCA for SVT significantly reduces ionising radiation dose and fluoroscopy time. The use of NFS also decreases the procedure time of patients undergoing 
left-sided AVRT ablation. The benefits of NFS for both patients and medical staff in reducing the adverse effects of radiation should not be undervalued.

\section{REFERENCES}

1. Blomström-Lundqvist $C$, Scheinman MM, Aliot EM, et al; ACC/AHA ESC guidelines for the management of patients with supraventricular arrhythmias--executive summary: a report of the American College of Cardiology/American Heart Association Task Force on Practice Guidelines and the European Society of Cardiology Committee for Practice Guidelines (Writing Committee to Develop Guidelines for the Management of Patients With Supraventricular Arrhythmias). Circulation 2003; 108:1871-909.

2. Rosenthal LS, Mahesh M, Beck TJ, et al. Predictors of fluoroscopy time and estimated radiation exposure during radiofrequency catheter ablation procedures. Am J Cardiol 1998; 82:451-8.

3. Razminia M, Manankil MF, Eryazici PL, et al. Nonfluoroscopic cathete ablation of cardiac arrhythmias in adults: feasibility, safety, and efficacy. J Cardiovasc Electrophysiol 2012; 23:1078-86.

4. Packer DL. Three-dimensional mapping in interventional electrophysiology: techniques and technology. J Cardiovasc Electrophysiol 2005; 16:1110-6.

5. Estner HL, Deisenhofer I, Luik A, et al. Electrical isolation of pulmonary veins in patients with atrial fibrillation: reduction of fluoroscopy exposure and procedure duration by the use of a non-fluoroscopic navigation system (NavX). Europace 2006; 8:583-7.

6. Stabile G, Scaglione M, del Greco M, et al. Reduced fluoroscopy exposure during ablation of atrial fibrillation using a novel electroanatomical navigation system: a multicenter experience. Europace 2012; 14:60-5

7. González-Torrecilla E, Arenal A, Quiles J, et al. [Non-fluoroscopic electroanatomical mapping (CARTO System) in the ablation of atrial tachycardias]. Rev Esp Cardiol 2004; 57:37-44. Spanish.

8. Earley MJ, Showkathali R, Alzetani M, et al. Radiofrequency ablation of arrhythmias guided by non-fluoroscopic catheter location: a prospective randomized trial. Eur Heart J 2006; 27:1223-9.

9. Kopelman HA, Prater SP, Tondato F, Chronos NA, Peters NS. Slow pathway catheter ablation of atrioventricular nodal re-entrant tachycardia guided by electroanatomical mapping: a randomized comparison to the conventional approach. Europace 2003; 5:171-4.

10. Kirchhof P, Loh P, Eckardt L, et al. A novel nonfluoroscopic catheter visualization system (LocaLisa) to reduce radiation exposure during catheter ablation of supraventricular tachycardias. Am J Cardiol 2002; 90:340-3.

11. Alvarez M, Tercedor L, Almansa I, et al. Safety and feasibility of catheter ablation for atrioventricular nodal re-entrant tachycardia without fluoroscopic guidance. Heart Rhythm 2009; 6:1714-20.

12. Kesek M, Wallenius N, Rönn F, Höglund N, Jensen S. Reduction of fluoroscopy duration in radiofrequency ablation obtained by the use of a non-fluoroscopic catheter navigation system. Europace 2006; 8:1027-30.

13. Casella M, Pelargonio G, Dello Russo A, et al. "Near-zero" fluoroscopic exposure in supraventricular arrhythmia ablation using the EnSite NavX ${ }^{\mathrm{TM}}$ mapping system: personal experience and review of the literature. J Interv Card Electrophysiol 2011; 31:109-18.

14. Bedetti G, Botto N, Andreassi MG, et al. Cumulative patient effective dose in cardiology. Br J Radiol 2008; 81:699-705.

15. Fazel R, Krumholz HM, Wang Y, et al. Exposure to low-dose ionizing radiation from medical imaging procedures. N Engl J Med 2009; 27; 361:849-57.

16. Venneri L, Rossi F, Botto N, et al. Cancer risk from professional exposure in staff working in cardiac catheterization laboratory: insights from the National Research Council's Biological Effects of Ionizing Radiation VII Report. Am Heart J 2009; 157:118-24.

17. National Council on Radiation Protection and Measurements. Ionizing Radiation Exposure of the Population of the United States: Recommendations of the National Council on Radiation Protection and Measurements. 2009 Mar. Report no: 160. 